\title{
Intravenous high mobility group box 1 upregulates the expression of HIF-1 $\alpha$ in the myocardium via a protein kinase B-dependent pathway in rats following acute myocardial ischemia
}

\author{
HENG-CHEN YAO ${ }^{1,2}$, MIN ZHOU ${ }^{2}$, YAN-HONG ZHOU ${ }^{2}$, LAN-HUA WANG ${ }^{2}$, DE-YONG ZHANG ${ }^{2}$, \\ QIAN-FENG HAN ${ }^{2}$, TAO LIU ${ }^{2}$, LEI WU ${ }^{2}$, KE-LI TIAN ${ }^{3}$ and MEI ZHANG ${ }^{1}$ \\ ${ }^{1}$ Department of Cardiology, Qilu Hospital of Shandong University School of Medicine, Jinan, Shandong 250012; \\ ${ }^{2}$ Department of Cardiology, Liaocheng People's Hospital and Clinical School of Taishan Medical University, \\ Liaocheng, Shandong 252000; ${ }^{3}$ Department of Biochemistry and Molecular Biology, \\ Shandong University School of Medicine, Jinan, Shandong 250012, P.R. China
}

Received January 31, 2015; Accepted November 10, 2015

DOI: $10.3892 / \mathrm{mmr} .2015 .4648$

\begin{abstract}
The effects of intravenous high mobility group box 1 (HMGB1) on myocardial ischemia/reperfusion (I/R) injury remains to be elucidated. The purpose of the present study was to investigate the effects of intravenous HMGB1 on the expression of hypoxia inducible factor- $1 \alpha$ (HIF-1 $\alpha$ ) in the myocardium of rats following acute myocardial ischemia, and to examine the effects of intravenous HMGB1 on myocardial I/R injury. Male Wistar rats were divided into the following groups: Sham operation group $(n=10)$, a group exposed to ischemia for $30 \mathrm{~min}$ and reperfusion for $4 \mathrm{~h}$ (I/R group) as a control ( $\mathrm{n}=10)$, an HMGB group, in which 100 ng/kg HMGB was administered intravenously $30 \mathrm{~min}$ prior to ischemia $(n=10)$, an LY group, in whic LY294002, an inhibitor of phosphoinositide 3-kinase (PI3K), was administered intravenously $(0.3 \mathrm{mg} / \mathrm{kg}) 40 \mathrm{~min}$ prior to ischemia $(\mathrm{n}=10)$, and the HMGB1+LY group, in which HMGB1 (100 ng/kg) and LY294002 (0.3 mg/kg) were administered intravenously $30 \mathrm{~min}$ and $40 \mathrm{~min}$ prior to ischemia, respectively $(\mathrm{n}=10)$. The serum levels of cardiac troponin I (cTnI) and tumor necrosis factor- $\alpha$ (TNF- $\alpha)$, and myocardial infarct size were measured. The expression levels of phosphorylated Akt and HIF-1 $\alpha$ were investigated using western blot analyses. The results showed
\end{abstract}

Correspondence to: Professor Ke-Li Tian, Department of Biochemistry and Molecular Biology, Shandong University School of Medicine, 44 Wenhua Xi Road, Jinan, Shandong 250012, P.R. China

E-mail: tiankeli@sdu.edu.cn

Professor Mei Zhang, Department of Cardiology, Qilu Hospital of Shandong University School of Medicine, 44 Wenhua Xi Road, Jinan, Shandong 250012, P.R. China

E-mail: lctougao@126.com

Key words: high mobility group box 1, acute myocardial ischemia, ischemia reperfusion injury, hypoxia inducible factor- $1 \alpha$, rats that pre-treatment with HMGB1 significantly decreased serum levels of cTnI, and TNF- $\alpha$, and reduced myocardial infarct size following $4 \mathrm{~h}$ reperfusion (all $\mathrm{P}<0.05$ ). HMGB1 also increased the expression levels of HIF-1 $\alpha$ and p-Akt induced by $\mathrm{I} / \mathrm{R}$ $(\mathrm{P}<0.05)$. LY294002 was found to eliminate the effects of intravenous HMGB1 on myocardial I/R injury $(\mathrm{P}<0.05)$. These results suggest that intravenous pre-treatment with HMGB1 may exert its cardioprotective effects via the upregulation of the myocardial expression of HIF-1 $\alpha$, which may be regulated by the PI3K/Akt signaling pathway, in rats following acute myocardial I/R.

\section{Introduction}

Acute myocardial infarction is a primary contributor to rates of mortality and morbidity, which has become a severe health problem worldwide (1). When acute myocardial infarction occurs, rapid reperfusion by either percutaneous coronary intervention or thrombolytic therapy is important for salvaging myocardial tissue from inevitable necrosis, and to decrease infarct size (IS). Paradoxically, reperfusion itself is frequently associated with the exacerbation of tissue injury and profound inflammatory responses, termed ischemia reperfusion (I/R) injury $(2,3)$. In addition, the exposure of a single organ to $\mathrm{I} / \mathrm{R}$ may subsequently result in inflammatory activation in other organs, eventually leading to multi-organ failure and markedly increasing rates of mortality and morbidity (4). Therefore, the alleviation of myocardial I/R injury is an important strategy in the management of acute myocardial ischemia.

Hypoxia inducible factor- $1 \alpha$ (HIF-1 $\alpha$ ) is a central transcription factor that is key in cellular adaption to hypoxia and ischemia, which enables cells to survive and differentiate in low oxygen conditions (5) HIF-1 $\alpha$ is upregulated in hypoxic condition by driving the expression of $>100$ genes (6). HIF-1 $\alpha$ also assists in restoring oxygen homeostasis by inducing glycolysis, erythropoiesis and angiogenesis (7). It is important in cardioprotection following I/R injury (8). Our previous study revealed that there is an association between increased myocardial expression levels of HIF-1 $\alpha$ and cardioprotective 
effects in rats following acute myocardial ischemia (9). Therefore, HIF-1 $\alpha$ modulation may reduce tissue injury during acute myocardial ischemia.

High mobility group box 1 (HMGB1), a ubiquitous and abundant nuclear protein, can either be passively released into the extracellular milieu in response to necrotic signals, or actively secreted in response to inflammatory signals $(10,11)$. HMGB1 is widely distributed in the liver, brain, spleen, lung, heart, kidney and lymphatic tissue (12). The first 40 peptide segments of the B-box can induce the production of tumor necrotic factor- $\alpha$ (TNF- $\alpha$ ) and interleukin (IL)-6 (13).

As a pro-inflammatory cytokine, HMGB1 is important in several cardiovascular diseases, including atherosclerosis, myocardial I/R injuries, heart failure and myocardial infarction (14-18). Clinical studies have shown that circulating levels of HMGB1 correlate with the severity of coronary artery disease, and may be a potential and independent predictor of cardiovascular mortality rates in patients with unstable angina/non ST segment elevation myocardial infarction $(18,19)$. Extracellular HMGB1 not only represents an optimal 'necrotic marker', selected by the innate immune system to recognize tissue damage and initiate reparative responses, but acts as a potent pro-inflammatory cytokine, which contributes to the pathogenesis of diverse inflammatory effects and promotes I/R-induced myocardial injury $(20,21)$.

Previously, it has been reported that the exogenous administration of HMGB1 following myocardial infarction or acute global I/R leads to the recovery of left ventricular function through the regeneration of cardiomyocytes. However, once the dose of HMGB1 increases, it can only inhibit inflammatory reactions, rather than improve the recovery of left ventricular function (22-24). Furthermore, previous studies have investigated HMGB1 through direct myocardial injection in various animal models. However, to date, the effects of intravenously infused HMGB1 on I/R injury in the myocardium, and the underlying mechanisms, remain to be fully elucidated.

The aim of the present study was to investigate the effects of intravenous HMGB1 on the expression of HIF-1 $\alpha$ in the myocardium of rats following acute myocardial ischemia. In addition, the effects of intravenous HMGB1 on myocardial ischemia reperfusion injury and the underlying mechanisms were evaluated.

\section{Materials and methods}

Animal groups. All experiments were performed in accordance with the Guide for the Care and Use of Laboratory Animals, published by the US National Institutes of Health (no. 85-23, revised 1996) (25), and were approved by the Institutional Review Board of Liaocheng People's hospital (Liaocheng, China). Male Wistar rats, aged 8-11 weeks $(n=50$; body weight, 250-300 g) were obtained from the experimental laboratory of Shandong Lukang Co., Ltd. (Jining, China). The rats were maintained in a controlled environment under a $12-\mathrm{h}$ light/dark cycle (humidity, $55 \pm 5 \%$; temperature $22 \pm 1^{\circ} \mathrm{C}$ ) with free access to food and water (provided by the experimental laboratory of Shandong Lukang, Ltd. (Shandong, China). The rats were randomly divided into five groups, each containing 10 animals: i) sham operation (sham) group; ii) ischemia reperfusion (I/R) group; iii) I/R rats pre-treated intravenously with $100 \mathrm{ng} / \mathrm{kg}$ recombinant HMGB1 (R\&D Systems, Inc., Minneapolis, MN, USA) $30 \mathrm{~min}$ prior to ischemia (HMGB1 group); (iv) I/R rats pre-treated intravenously with LY294002 (Sigma-Aldrich, St. Louis, MO, USA), an inhibitor of phosphoinositide 3-kinases (PI3K), at a dose of $0.3 \mathrm{mg} / \mathrm{kg}$ (LY group); (v) I/R rats pre-treated with HMGB1 (100 ng/kg) and LY294002 $(0.3 \mathrm{mg} / \mathrm{kg})$ intravenously 30 and $40 \mathrm{~min}$ prior to ischemia, respectively) (HMGB1+LY group). HMGB1 (dissolved in phosphate buffered saline) and LY (dissolved in dimethyl sulfoxide) were administered intravenously in the tail vein, in a $0.5-\mathrm{ml}$ volume. The sham group was treated with an intravenous injection of normal saline $(0.5 \mathrm{ml})$.

Animal model. The rat I/R model was established according to methods previously reported (26). All the I/R rats were subjected to left anterior descending coronary artery (LAD) occlusion via a suture for $30 \mathrm{~min}$, followed by reperfusion for $4 \mathrm{~h}$. Following general anesthesia via intraperitoneal injection of sodium pentobarbital (60 mg/kg; Sigma-Aldrich), the trachea was cannulated for artificial ventilation with room air, at a rate of 55 breaths $/ \mathrm{min}$. The body temperature of the rats was maintained at $37 \pm 0.50^{\circ} \mathrm{C}$ using an electrical heating pad (Ruiwode Life Science Inc., Shenzhen, China). Lead II of an electrocardiogram (ECG) was monitored using stainless needle electrodes, which were attached to limbs. The ECG was recorded and analyzed using an ECG-6511 data acquisition system (Guangdian Medical Device Co. Ltd., Shanghai, China). In the sham group, the suture was placed at the origin of the LAD, however, no complete ligation of the artery was performed.

Biochemical analysis. Blood samples $(1 \mathrm{ml})$ were obtained from the femoral vein following reperfusion for $4 \mathrm{~h}$, and were centrifuged at $1,400 \mathrm{x}$ for $10 \mathrm{~min}$ at $4^{\circ} \mathrm{C}$. The sera were obtained and then frozen at $-80^{\circ} \mathrm{C}$ until future analyses. Serum levels of TNF- $\alpha$ were determined, according to the manufacturer's protocol, using a rat TNF- $\alpha$ enzyme-linked immunosorbent assay (ELISA) kit (Shanghai Huiying Biotechnology Ltd., Co., Zhongshan, China). Serum levels of cardiac troponin (c-TnI) and IL-6 were detected using rat CTn-I (Lengton Bioscience Co., Ltd., Shanghai, China) and IL-6 (Jingmei Biotech Co., Ltd., Shenzhen, China) ELISA kits, respectively.

Measurement of myocardial activities of malondialdehyde (MDA) and superoxide dismutase (SOD) (27). The hearts were harvested from the rats and washed with normal saline. Subsequently, $0.5 \mathrm{~g}$ of the ischemic heart tissue was ground at $0-4^{\circ} \mathrm{C}$, following which the myocardial homogenate was centrifuged at 2,500 x g for $30 \mathrm{~min}$. The supernatants $(\sim 0.4 \mathrm{~g})$ were harvested and stored at $-80^{\circ} \mathrm{C}$ until MDA concentration and SOD activity assays were performed. A thiobarbituric acid reactive substance assay (DTBA-100; Bioassay Systems LLC, Hayward, CA, USA) was used to measure the levels of MDA, by measuring the absorbance value at a wavelength of $532 \mathrm{~nm}$ using a microplate reader (SP-Max 2300A; Molecular Devices, LLC, Sunnyvale, CA, USA). A xanthine oxide method was used to determine SOD activity, by measuring the absorbance value at a wavelength of $550 \mathrm{~nm}$. An MDA Assay kit and an SOD Assay kit (Nanjing Jiancheng Bioengineering Co., Ltd., Nanjing, China) were used to measure MDA concentration and 
Table I. Serum levels of IL-6, TNF- $\alpha$, cTnI, SOD and MDA, and IS.

\begin{tabular}{lccccc}
\hline Variable & Sham & I/R & HMGB1 & LY & HMGB1+LY \\
\hline IL-6 $(\mathrm{pg} / \mathrm{ml})$ & $160.48 \pm 12.03$ & $385.44 \pm 15.34^{\mathrm{a}}$ & $215.66 \pm 18.11^{\mathrm{a}, \mathrm{b}}$ & $376.86 \pm 17.05^{\mathrm{a}, \mathrm{c}, \mathrm{d}}$ & $355.32 \pm 20.53^{\mathrm{a}, \mathrm{c}}$ \\
TNF- $\alpha(\mathrm{pg} / \mathrm{ml})$ & $13.72 \pm 5.18$ & $76.98 \pm 6.89^{\mathrm{a}}$ & $52.53 \pm 3.87^{\mathrm{a}, \mathrm{b}}$ & $77.22 \pm 5.01^{\mathrm{a}, \mathrm{c}, \mathrm{d}}$ & $70.32 \pm 4.99^{\mathrm{a}, \mathrm{c}}$ \\
cTnI $(\mu \mathrm{g} / \mathrm{l})$ & $0.09 \pm 0.22$ & $73.47 \pm 8.11^{\mathrm{a}}$ & $47.33 \pm 3.16^{\mathrm{a}, \mathrm{b}}$ & $70.42 \pm 7.08^{\mathrm{a}, \mathrm{c}, \mathrm{d}}$ & $63.56 \pm 4.71^{\mathrm{a}, \mathrm{c}}$ \\
SOD $(\mathrm{u} / \mathrm{mg})$ & $142.18 \pm 27.67$ & $64.67 \pm 19.91^{\mathrm{a}}$ & $101.79 \pm 31.6^{\mathrm{a}, \mathrm{b}}$ & $64.42 \pm 13.37^{\mathrm{a}, \mathrm{c}, \mathrm{d}}$ & $75.13 \pm 16.65^{\mathrm{a}, \mathrm{c}}$ \\
MDA $(\mathrm{nmol} / \mathrm{mg})$ & $1.27 \pm 0.23$ & $8.45 \pm 0.47^{\mathrm{a}}$ & $3.55 \pm 0.67^{\mathrm{a}, \mathrm{b}}$ & $8.56 \pm 0.96^{\mathrm{a}, \mathrm{c}, \mathrm{d}}$ & $8.14 \pm 0.76^{\mathrm{a}, \mathrm{c}}$ \\
IS $(\%)$ & 0 & $55.18 \pm 4.43^{\mathrm{a}}$ & $32.89 \pm 2.12^{\mathrm{a}, \mathrm{b}}$ & $57.60 \pm 3.59^{\mathrm{a}, \mathrm{c}}$ & $49.73 \pm 4.78^{\mathrm{a}, \mathrm{c}}$
\end{tabular}

Data are presented as the mean \pm standard deviation. ${ }^{\mathrm{a}} \mathrm{P}<0.01$, vs. sham; ${ }^{\mathrm{b}} \mathrm{P}<0.01$, vs. I/R; ${ }^{\mathrm{C}} \mathrm{P}<0.01$, vs. HMGB1; ${ }^{\mathrm{d}} \mathrm{P}<0.01 \mathrm{vs}$. HMGB1+LY. $/ \mathrm{R}$, ischemia/reperfusion; HMGB1, high mobility group box 1; LY, LY29002; IL-6, interleukin-6; TNF- $\alpha$, tumor necrosis factor- $\alpha$; cTnI, cardiac troponin I; SOD, superoxide dismutase; MDA, malondialdehyde; IS, infarct size.

SOD activity, respectively, according to the manufacturer's protocol. The conditions of the assays were as follows: MDA, $90-100^{\circ} \mathrm{C}$ for $50 \mathrm{~min}$; SOD, $37^{\circ} \mathrm{C}$ for $20 \mathrm{~min}$.

Histological analysis of the heart. The rats were sacrificed by decapitation, the hearts were immediately removed and fixed in $10 \%$ formalin for $60 \mathrm{~min}$ at room temperature, and then for $24 \mathrm{~h}$ at $4^{\circ} \mathrm{C}$. Subsequently, the samples were washed and stored in $70 \%$ ethanol at $4^{\circ} \mathrm{C}$, prior to being embedded in paraffin wax (Leica Biosystems Richmond Inc., Richmond, IL, USA) and sectioning. For MDA and SOD measurement, there were five rats per group, and five rats underwent histological analysis. The paraffin sections $(5 \mu \mathrm{m})$ were stained with hematoxylin and eosin, using a Tissue-Tek ${ }^{\circledR}$ DRS $^{\text {TM }} 2000$ (all obtained from Sakura Finetek Japan Co., Ltd., Tokyo, Japan). Myocardiocyte and tissue morphology were examined under a digitalized microscope (Eclipse 80i; Nikon Corporatin, Tokyo, Japan).

Assessment of infarct size (IS). The IS was assessed using 2,3,5-triphenyltetrazolium chloride (TTC; Sigma-Aldrich) staining methods, as previously reported (26). Following reperfusion for $4 \mathrm{~h}$, the LAD was occluded again on five rats from each group to assess the IS, and $1 \mathrm{ml} 2.0 \%$ Evans blue dye (Sigma-Aldrich) was injected via the chest aorta. The entire heart was excised, rinsed of excess blue dye with normal saline, and the right and left atria were removed. The left ventricle was deep frozen at $-80^{\circ} \mathrm{C}$. The frozen left ventricle was then sliced horizontally to yield five slices between the apex and base. The slices were incubated in $1 \%$ TTC for 15 min at $37^{\circ} \mathrm{C}$. The impaired myocardium was stained red, the infarcted myocardium was stained white and the normal myocardium was stained blue. Images of the slices were captured using a Nikon D90 digital camera (Nikon Corporation, Tokyo, Japan). The borders of the infarct, ischemic and nonischemic areas of the heart images were traced and measured using Image-Pro Plus 3.0 (Media Cybernetics, Silver Spring, MD, USA). Infarct size was calculated as a percentage of the risk area (infarct size / risk area).

Western blot analysis. The protein expression levels of HIF-1 $\alpha$ and p-Akt were analyzed using western blot analysis. Myocardial tissues were dissected from each of the treatment groups, and the rodent tissue homogenates were prepared in Tris-HCl buffer
(pH 7.5), containing $150 \mathrm{mM} \mathrm{NaCl}, 1 \%$ NP-40, 1 mM EDTA, $1 \mu \mathrm{g} / \mathrm{ml}$ leupeptin, $1 \mu \mathrm{g} / \mathrm{ml}$ pepstatin, $3.8 \mu \mathrm{g} / \mathrm{ml}$ aprotinin, $1 \mathrm{mM}$ PMSF, $1 \mathrm{mM} \mathrm{Na} \mathrm{VO}_{4}$ and $2 \mathrm{mM} \mathrm{NaF}$ (all Beyotime Institute of Biotechnology, Haimen, China). The extracts were clarified by centrifugation at $4^{\circ} \mathrm{C}(14,000 \mathrm{x} \mathrm{g}$ for $20 \mathrm{~min})$. Supernatants were collected and eluted with SDS sample buffer (Beyotime Institute of Biotechnology), and the proteins were resolved using 10\% SDS-PAGE (Beyotime Institute of Biotechnology). Bicinchoninic acid protein assay reagent (Sigma-Aldrich) was used to determine the protein expression levels. Proteins were separated by PAGE and transferred onto polyvinilidene fluoride membranes (Bio-Rad Laboratories, Inc., Hercules, CA, USA) with $50 \mu \mathrm{g}$ of lysate per lane. The membranes were blocked with $5 \%$ non-fat milk in $400 \mathrm{ml}$ Tris-buffered saline (1X) with $200 \mu 1$ Tween-20 (Beyotime Institute of Biotechnology) at room temperature for $60 \mathrm{~min}$. The membranes were incubated with the following primary antibodies at $4^{\circ} \mathrm{C}$ for $24 \mathrm{~h}$ : Rabbit anti-rat HIF-1 $\alpha$ polyclonal antibody (cat. no. sc-10790; Santa Cruz Biotechnology, Inc., Santa Cruz, CA, USA; 1:2,000), rabbit anti-rat $\beta$-actin monoclonal antibody (cat. no. ab119716; Abcam, Cambridge, UK; 1:5,000), rabbit anti-rat p-Akt monoclonal antibody (cat. no. 4060; Cell Signaling Technology, Inc., Beverly, MA, USA; 1:1,000) and rabbit anti-rat t-Akt monoclonal antibody (cat. no. 4691; Cell Signaling Technology, Inc.; 1:1,000). The membranes were then incubated with secondary antibody, IRDye680 goat anti-rabbit IgG (cat. no. 926-68071, 925-68071; LI-COR Biosciences, Lincoln, NE, USA; 1:2,000) at room temperature for $2 \mathrm{~h}$. Following incubation, the membranes were exposed to chemiluminescence and a ChemiDoc imaging system (Bio-Rad Laboratories, Inc.,) to analyze the protein bands. The relative content of each sample was repeated at least three times.

Statistical analysis. Data are expressed as the mean \pm standard deviation or percentages, where appropriate. SAS 6.12 software (SAS Institute, Inc., Cary, NC, USA) was used for statistical analyses. One way analysis of variance was used to compare the means between groups. $\mathrm{P}<0.05$ was considered to indicate a statistically significant difference.

\section{Results}

Serum levels of $c-T n I$ and TNF- $\alpha$. As shown in Table I, serum levels of IL-6, c-TnI and TNF- $\alpha$ in the I/R group were 

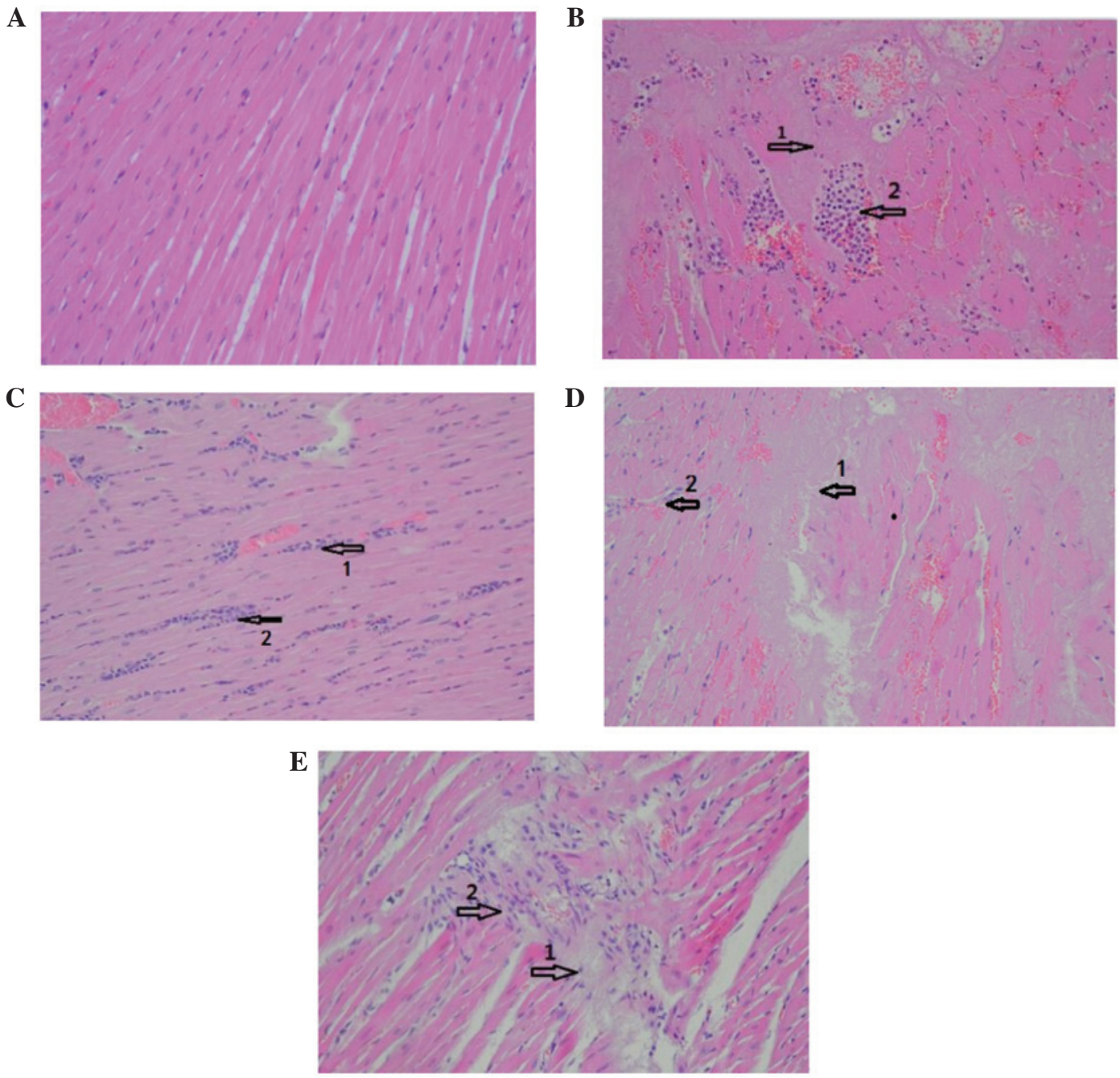

Figure 1. Histopathological changes in myocardial tissue, determined using hematoxylin and eosin staining (magnification, $\mathrm{x} 400$ ). (A) Sham group; myocardial fibers were arranged regularly with clear boundaries and no inflammatory infiltration. (B) I/R group; focal myocardial damage with uneven staining was observed. Morphological changes in affected cardiomyocytes primarily comprised different degrees of swelling, necrosis, myocytolysis and myofibrillar loss. Myocardial fibers were disrupted and arranged irregularly (arrow 1). Infarction foci were infiltrated with numerous neutrophils (arrow 2). (C) HMGB group; myocardial fibers were arranged relatively regularly (arrow 1). Infiltration of numerous neutrophils was observed (arrow 2). (D) LY group; myocardial fibers were disrupted and dissolved, and stripes had disappeared (arrow 1). Numerous inflammatory cells infiltrated the area surrounding the infarction foci and capillaries (arrow 2). (E) HMGB1+LY group; myocardial fibers were arranged irregularly, a number being dissolved and disrupted. The number of cardiomyocytes were reduced and intermuscular spaces widened (arrow 1). Infarction foci and capillaries were surrounded by infiltration of inflammatory cells (arrow 2). I/R, ischemia/reperfusion;HMBG, high mobility group box 1; LY, LY294002.

significantly higher, compared with those in the sham group $(\mathrm{P}<0.01)$. Pre-treatment with HMGB1 significantly decreased serum levels of IL-6, c-TnI and TNF- $\alpha$, compared with the I/R group $(\mathrm{P}<0.01)$. However, compared with the HMGB1 group, the decreased serum levels of IL-6, c-TnI and TNF- $\alpha$ in the LY and LY+HMGB1 groups were significantly reversed $(\mathrm{P}<0.01)$. Serum levels of IL-6, c-TnI and TNF- $\alpha$ in the LY group were significantly higher, compared with those in the LY+HMGB1 group $(\mathrm{P}<0.01)$.

MDA levels and SOD activity. The results of the MDA and SOD anaylses, shown in Table I, indicated that the myocardial level of MDA in the I/R group was significantly increased, whereas the activity of SOD was significantly decreased, compared with the sham group $(\mathrm{P}<0.0)$. The increase in the level of MDA and reduction of SOD activity were significantly inhibited by HMGB1 pretreatment $(\mathrm{P}<0.01)$. However, the decreased levels of MDA and increased SOD activities were reversed significantly in the LY and LY+HMGB1 groups, compared with the HMGB1 group $(\mathrm{P}<0.01)$. The myocardial level of MDA increased and SOD activity decreased significantly in the LY group, compared with the LY+HMGB1 group $(\mathrm{P}<0.01)$.

Histological analysis of the heart. In the sham group, myocardial fibers were arranged regularly with clear boundaries, and no inflammatory infiltration was present (Fig. 1A). In the I/R group (Fig. 1B), morphological changes in the affected cardiomyocytes primarily comprised different degrees of swelling, necrosis, myocytolysis and myofibrillar loss. The myocardial fibers were disrupted and arranged irregularly. The foci of myocardial infarction were infiltrated with an increased number of neutrophils. In the HMGB group (Fig. 1C), the myocardial fiber arrangement was relatively regular. Infiltration of an increased number of neutrophils were also observed. In the LY group (Fig. 1D), myocardial fibers were disrupted and 

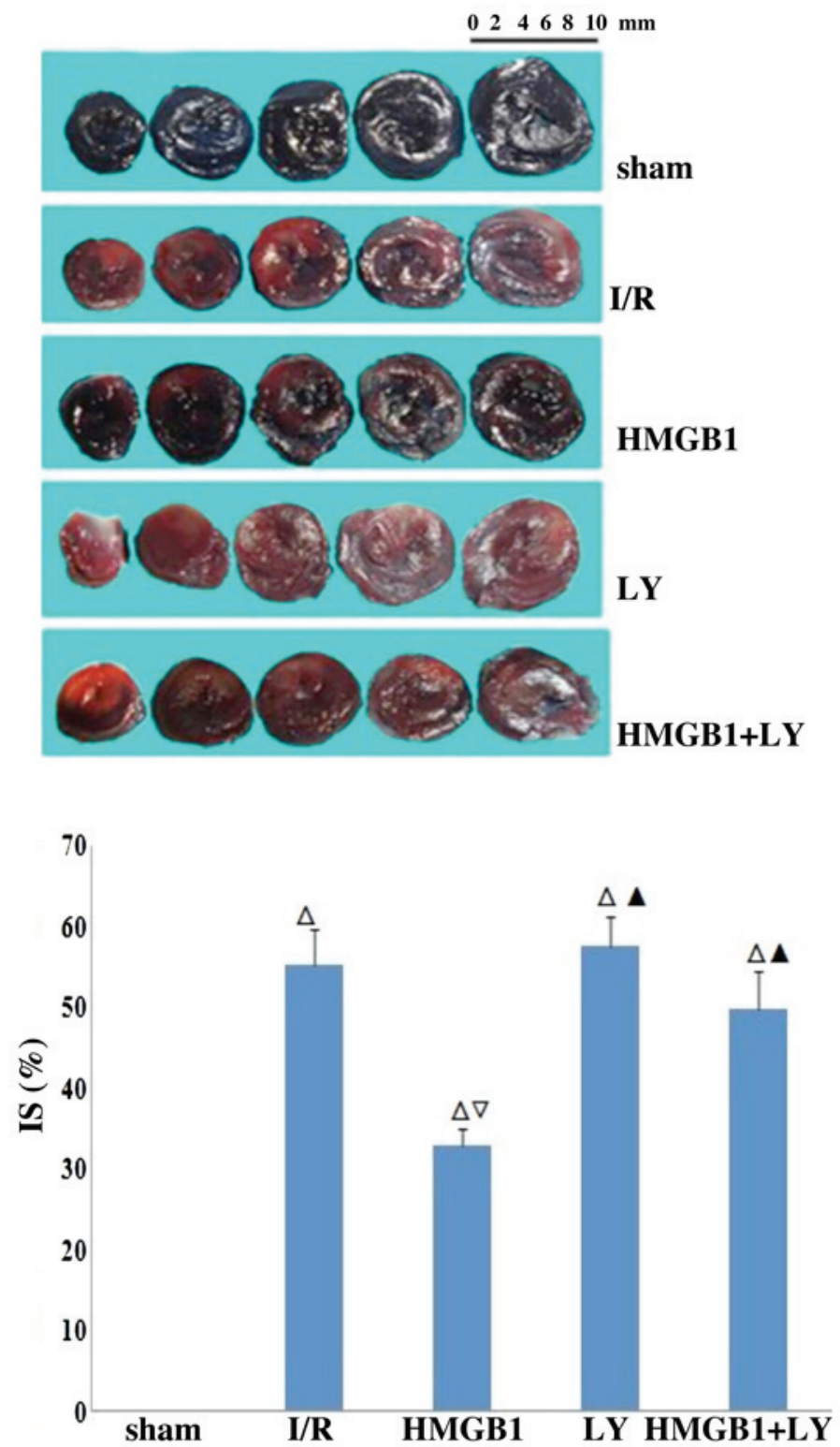

Figure 2. IS in the treatment groups. 2,3,5-triphenyltetrazolium chloride-Evans blue staining was used to determine the area of the myocardial infarction. Normal myocardium was stained blue, ischemic myocardium was stained a brick red color and infarcted myocardium stained white. IS, infarct size; I/R, ischemia/reperfusion; LY, LY294002; HMGB1, high mobility group box $1 .{ }^{\wedge} \mathrm{P}<0.05$ vs. sham group; ${ }^{\mathrm{P}}<0.05$ vs. I/R group; ${ }^{\wedge} \mathrm{P}<0.05$ vs. HMGB1 group.

dissolved, and the stripes were no longer visible. An increased number of inflammatory cells were observed to have infiltrated surrounding the myocardial infarction foci and capillaries. In the HMGB1+LY group (Fig. 1E), myocardial fibers were arranged irregularly, and were dissolved and disrupted in places. The number of myocardiocytes were reduced and the intermuscular spaces were widened. Myocardial infarction foci and capillaries were surrounded by inflammatory cell infiltration.

Comparison of IS in each group. As shown in Fig. 2 and Table I, the IS in the I/R group was significantly higher, compared with those in the sham group $(\mathrm{P}<0.01)$. The IS in the HMGB1-treated group was significantly decreased, compared with those in the I/R group $(\mathrm{P}<0.01)$. However, the IS was significantly increased in the LY group and LY+HMGB1, compared with the HMGB1-treated group $(\mathrm{P}<0.01)$.
Protein expression of HIF-1 $\alpha$. As shown in Fig. 3, the protein expression of HIF-1 $\alpha$ was significantly increased in the I/R group, compared with the sham group $(\mathrm{P}<0.05)$. Treatment with HMGB1 significantly increased the protein expression of HIF-1 $\alpha$, compared with the I/R group $(\mathrm{P}<0.05)$. However, the LY group and LY+HMGB group were found to have significantly lower protein expression levels of HIF-1 $\alpha$, compared with the HMGB group $(\mathrm{P}<0.05)$.

Protein expression of $p$-Akt. As shown in Fig. 4, the protein expression of p-Akt was significantly increased in the I/R group, compared with the sham group $(\mathrm{P}<0.05)$. Treatment with HMGB1 significantly increased the protein expression of p-Akt, compared with the I/R group $(\mathrm{P}<0.05)$. However the LY group and LY+HMGB group were found to have significantly lower protein expression levels of $\mathrm{p}$-Akt, compared with the HMGB group $(\mathrm{P}<0.05)$. 

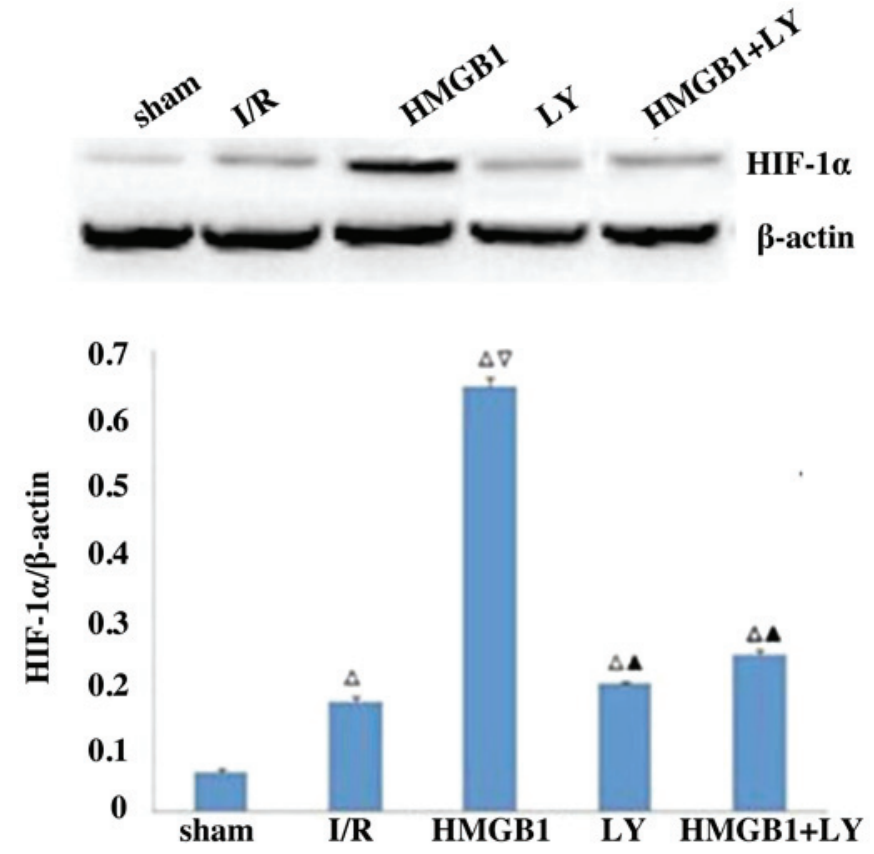

Figure 3. Protein expression of HMGB1. Data are expressed as the mean \pm standard deviation. ${ }^{\triangle} \mathrm{P}<0.05$ vs. sham group; ${ }^{\circ} \mathrm{P}<0.05$ vs. I/R group; $\triangle \mathrm{P}<0.05$ vs. HMGB1 group. I/R, ischemia/reperfusion; LY, LY294002; HMGB1, high mobility group box $1 ; \mathrm{HIF}-1 \alpha$, hypoxia inducible factor- $1 \alpha$.
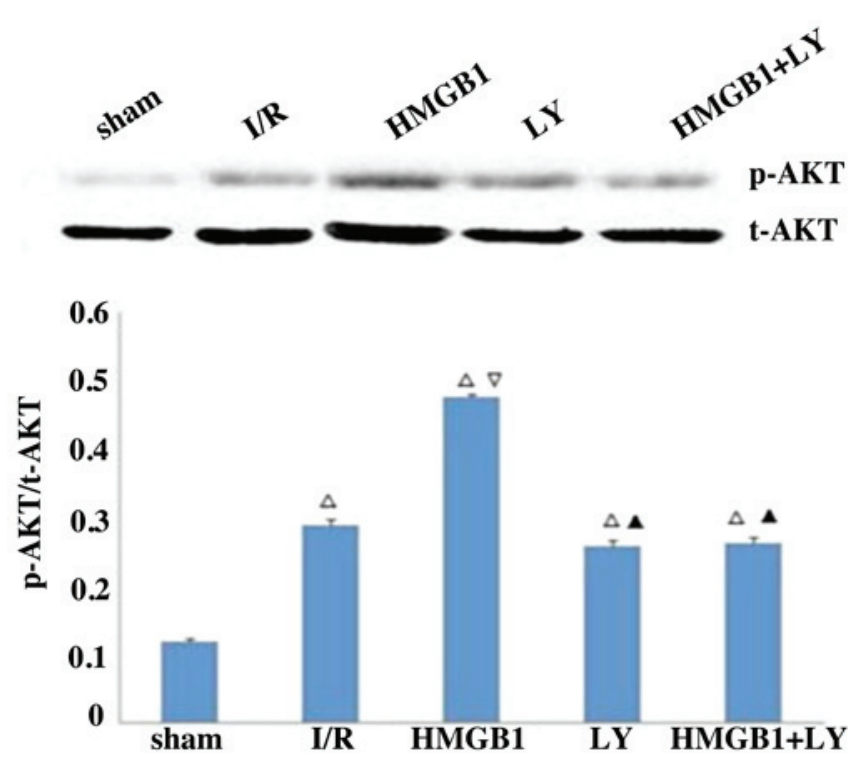

Figure 4. Protein expression of p-Akt. Data are expressed as the mean $\pm \mathrm{stan}$ dard deviation. ${ }^{\wedge} \mathrm{P}<0.05$ vs. sham group; ${ }^{\triangleright} \mathrm{P}<0.05$ vs. I/R group; ${ }^{\wedge} \mathrm{P}<0.05$ vs. HMGB1 group. I/R, ischmia/reperfusion; LY, LY294002; HMGB1, high mobility group box 1; p-Akt, phosphorylated Akt; t-Akt, total Akt.

\section{Discussion}

In the present study, the results demonstrated that: i) myocardial expression of HIF- $1 \alpha$ increased significantly in I/R rats; ii) pretreatment with HMGB1 led to significant anti-inflammatory effects, reducing infarct size and increasing the myocardial expression of HIF-1 $\alpha$; iii) cardioprotective effects induced by intravenous HMGB1 may be associated with enhancement of the protein expression of HIF-1 $\alpha$. This protective effect involved a reduction in oxidative stress, neutrophil infiltration and pro-inflammatory effects; iv) treatment with the PI3K inhibitor, LY294002, inhibited Akt phosphorylation and the cardioprotective effects of intravenous HMGB1. These results suggested that intravenous HMGB1 may alleviate myocardial $\mathrm{I} / \mathrm{R}$ injury through an increase in the protein expression of HIF-1 $\alpha$, involving the PI3K/Akt/HIF signaling pathway.

The rapid restoration of blood flow in the occluded coronary artery following acute myocardial ischemia is the most important aspect in protecting myocardial tissue from inevitable necrosis. However, the early opening of an occluded coronary artery may cause myocardial I/R injury $(2,3)$. Therefore, the attenuation of myocardial I/R injury is an important strategy in the treatment of acute myocardial ischemia. The present study hypothesized that HMGB1 pretreatment may protect heart against I/R injury, thus decreasing infarct size. I/R itself can cause sterile inflammation, which is characterized by the accumulation of inflammatory cells. The infiltration of leukocytes may, in turn, initiate an inflammatory response, which results in cardiomyocyte damage (2). These cells may also promote collateral tissue injury (28). Neutrophils are the predominant type of cells to aggregate in the ischemic myocardium following reperfusion, to mediate direct injury via the release of toxic products, including reactive oxygen species (ROS) and photolytic enzymes (29). It has also been reported that liver I/R injury is characterized by neutrophil recruitment and activation (30). Therefore, agents limiting the infiltration and activity of leukocytes, and inflammatory reaction have been suggested as a treatment strategy for attenuating myocardial I/R injury. In the present study, it was demonstrated that intravenous administration of HMGB1 prior to inducing myocardial ischemia inhibited the accumulation of leukocytes following myocardial I/R injury. Accordingly, pretreatment with HMGB1 attenuated I/R-induced necrosis, myocardial infarct size and the expression of proinflammatory cytokines, including TNF- $\alpha$. These results suggested that intravenous administration of HMGB1 may attenuate myocardial I/R injury through suppressing the recruitment of leukocytes and inhibiting the inflammatory reaction.

Several studies have shown that the process of myocardial $\mathrm{I} / \mathrm{R}$ injury is associated with the increased generation of ROS and oxidative stress $(31,32)$. Oxidative stress can modify proteins and phospholipids, resulting in lipid peroxidation and oxidation of thiol groups, which may lead to alteration of cell membrane configuration, and permeability and modification of various cellular proteins (33). I/R may cause arrhythmias, abnormality in gene expression, loss of adrenergic pathways and depression in contractile function (34). When the heart is pretreated with either cardiac subcellular organelles or various ROS-generating systems, similar changes to those mentioned above are found (3). However, when treated with SOD and catalase, the heart is protected from these changes (35). Therefore, it can be concluded that oxidative stress may result, at least in part, in these alterations in the myocardium during I/R. In the present study, it was found that HMGB1 reduced the myocardial content of MDA and increased the activity of SOD induced by I/R, whereas LY294002 eliminated these effects. It has been reported that ROS can directly injure the cell membrane and cause cell death during myocardial I/R (36). Furthermore, ROS-mediated apoptosis and necrosis can eventually be a determinant in infarct size (37). Therefore, 
HMGB1 may exert its cardioprotective through antioxidative stress in myocardial I/T injury, which may be regulated by the PI3K/Akt signaling pathway.

HIF-1 $\alpha$ is a heterodimeric DNA-binding complex, which is composed of oxygen sensitive, HIF-1 $\alpha$, and aryl hydrocarbon nuclear translocator, HIF-1 $\beta$ (38). HIF-1 $\alpha$ is continuously undergoing proteasomal degradation, resulting in a short half-life under normoxic conditions (39). By contrast, HIF-1 $\alpha$ can translocate to the nucleus and dimerize with HIF-1 $\beta$, as HIF-1 $\alpha$ evades proteasomal degradation under hypoxic conditions. The complex then binds to hypoxic response elements, driving the expression of $>100$ genes $(6,7)$. HIF- $1 \alpha$ regulates the cellular response to hypoxia. Studies have show that the expression of HIF-1 $\alpha$ increases significantly in different ischemic organs and tissues, including the retina (40), myocardium $(9,41)$ and nervous system $(42)$. It has also been reported that HIF-1 $\alpha$ can prevent hypoxia or I/R-induced myocardial injury in the heart $(8,43,44)$. In accordance with these previous studies, the present study found that basic fibroblast growth factor enhanced the myocardial mRNA expression of HIF-1 $\alpha$, thus decreasing infarct size and improving left ventricular function in rats following acute MI (9). As mentioned above, HIF-1 $\alpha$ overexpression confers protective effects against myocardial I/R injury by promoting the transcription of a number of cardioprotective genes, including those involved in glucose metabolism, mitochondrial function, cell apoptosis, erythropoietin, vascular endothelial growth factor, inducible nitric oxide synthase, hemeoxygenase, cardiotropin and resistance to oxidative stress $(45,46)$. Other mechanisms associated with the cardioprotective effects of HIF-1 $\alpha$ include prolyl hydroxylase 2 inhibition (8), the upregulation of cardiotrophin-1 (47) and the promotion of angiogenesis (48). A previous study showed that partial deficiency of HIF-1 $\alpha$ is associated with a complete loss of cardioprotection against I/R injury (49). Cardiomyocyte-specific HIF-1 $\alpha$ gene deletion causes reductions in contractility and vascularization, and alters the expression of multiple genes in the heart during normoxia (50).

The present study demonstrated that I/R significantly increased the myocardial expression of HIF-1 $\alpha$, and that the expression of HIF-1 $\alpha$ was markedly elevated by HMGB1. Furthermore, consistent with the increased expression of HIF-1 $\alpha$, the myocardial injury induced by I/R was inhibited by HMGB1. It was also found that intravenous HMGB1 decreased the levels of MDA and increased SOD activity in the I/R myocardium, which suggested that these changes may be located downstream of alterations in HIF-1 $\alpha$ overexpression. Thus, the results from the present study indicated that intravenous HMGB1 may exert its cardioprotective effects through increased myocardial expression of HIF-1 $\alpha$. However, the signaling pathway, which mediates the upregulation of HIF-1 $\alpha$ induced by HMGB1 in I/R myocardium, remains to be elucidated.

PI3K and the downstream effector Akt are a conserved family of signal transduction enzymes, which are involved in regulating cellular activation, inflammatory responses and apoptosis (51). Several previous studies have reported that the PI3K/Akt signaling pathway is key in myocardial protection against I/R injury (52-55). However, whether the PI3K/Akt pathway mediates the cardioprotective effects of intravenous HMGB1 remains to be fully elucidated. In the present study, it was found that HMGB1 markedly enhanced Akt phosphorylation, leading to the subsequent upregulation of HIF-1 $\alpha$ and attenuation of myocardial I/R injury. Treatment with LY294002 inhibited the HMGB1-induced expression of HIF-1 $\alpha$. Furthermore, the cardioprotective effects exerted by intravenous HMGB1 in an I/R rats model were eliminated by in vivo administration of PI3K inhibitor. Taken together, the present study hypothesized that HMGB1 upregulated the myocardial expression of HIF-1 $\alpha$, which was dependant, at least partially, on Akt phosphorylation, suggesting that the $\mathrm{PI} 3 \mathrm{~K} / \mathrm{Akt}$ signaling pathway may be involved in the cardioprotective effects of intravenous HMGB1 during myocardial I/R injury. Although the possibility of other signaling pathways also contributing to HMGB1-induced HIF-1 $\alpha$ expression, the results of the present study were consistent with previous studies, which suggested that the phosphorylation of Akt protected organs from I/R injury (53-58). Although multiple mechanisms are likely to be involved, the present study provided additional evidence that the protective effects of intravenous HMGB1 on myocardial I/R injury are, at least in part, regulated by the PI3K/Akt signaling pathway. The major mechanisms underlying these effects may include direct inhibition of leukocyte migration, production of ROS and inflammatory cytokines and increasing myocardial expression of HIF-1 $\alpha$, thus decreasing infarct size.

In conclusion, the present study involving an acute $\mathrm{I} / \mathrm{R}$ rat model demonstrated that the intravenous administration of HMGB1 was associated with a reduction in infarct size and increased myocardial expression levels of HIF-1 $\alpha$. The intravenous administration of HMGB1 may exert its cardioprotective effect by upregulating the protein expression of HIF-1 $\alpha$ in the ischemic myocardium via the PI3K/Akt signaling pathway.

\section{Acknowledgements}

This study was supported by the Natural Science Foundation of Shandong Province (grant no. ZR2013HL017), the Natural Science Foundation of Liaocheng City (grant no. 2012NS13) and the Science and Technology Developing Project of Liaocheng City (grant no. 2014GJH26).

\section{References}

1. Zweiter JL and Talukder MA: The role of oxidants and free radicals in reperfusion injury. Cardiolvasc Res 70: 181-190, 2006.

2. Yellon DM and Hausenloy DJ: Myocardial refusion injury. N Engl J Med 357: 1121-1135, 2007.

3. Eltzschig HK and Eckle T: Ischemia and reperfusion-from mechnism to translation. Nat Med 17: 1391-1401, 2011.

4. Park SW, Kim M, Brown KM, D'Agati VD and Lee HT: Paneth cell-derives IL-17A causes multiorgan dysfunction after hepatic ischemia and reperfusion injury. Hepatology 53: 1662-1675, 2011.

5. Maes C, Carmeliet G and Schipani E: Hypoxia-driven pathways in bone development, regeneration and disease. Nat Rev Rheumatol 8: 358-366, 2012.

6. Kasivisvanathan V, Shalhoub J, Lim CS, Shepherd AC, Thapar A and Davies AH: Hypoxia-inducible factor-1 in arterial disease: A putative therapeutic target. Curr Vasc Pharmacol 9: 333-349, 2011.

7. Weidemann A and Johnson RS: Biology of HIF-1alpha. Cell Death Differ 15: 621-627, 2008.

8. Poynter JA, Manukyan MC, Wang Y, Brewster BD, Herrmann JL, Weil BR, Abarbanell AM and Meldrum DR: Systemic pretreatment with dimethyloxalylglycine increases myocardial HIF- $1 \alpha$ and VEGF production and improves functional recovery after acute ischemia/reperfusion. Surgery 150: 278-283, 2011 
9. Yao HC, Liu T, Meng XY, Han QF, Zhang M and Wang LX: Effect of basic fibroblast growth factor on the myocardial expression of hypoxia-inducible factor- $1 \alpha$ and vascular endothelial growth factor following acute myocardial infarction. Heart Lung Circ 22: 946-951, 2013.

10. Lakhan SE, Kirchgessner A and Hofer M: Inflammatory mechnisms in ischemic stroke: Therapeutic approaches. J Transl Med 7: 97, 2009.

11. Oozawa S, Mori S, Kanke T, Takahashi H, Liu K, Tomono Y, Asanuma M, Miyazaki I, Nishibori M and Sano S: Effects of HMGB1 on ischemia-reperfusion injury in the rat heart. Circ J 72: 1178-1184, 2008.

12. Deneke SM and Fanburg BL. Normobaric oxygen toxicity of the lung. N Engl J Med 303: 76-86, 1980.

13. Li J, Kokkola R, Tabibzadeh S, Yang R, Ochani M, Qiang X, Harris HE, Czura CJ, Wang H, Ulloa L, et al. Structural basis for the proinflammatory cytokine activity of high mobility group box 1. Mol Med 9: 37-45, 2003.

14. Kohno T, Anzai T, Naito K, Miyasho T, Okamoto M, Yokota H, Yamada S, Maekawa Y, Takahashi T, Yoshikawa T, et al: Role of high-mobility group box 1 protein in post-infarction healing process and left ventricular remodelling. Cardiovasc Res 81: 565-573, 2009.

15. Yan XX, Lu L, Peng WH, Wang LJ, Zhang Q, Zhang RY, Chen QJ and Shen WF: Increased serum HMGB1 level is associated with coronary artery disease in nondiabetic and type 2 diabetic patients. Atherosclerosis 205: 544-548, 2009.

16. Avalos AM, Kiefer K, Tian J, Christensen S, Shlomchik M, Coyle AJ and Marshak-Rothstein A: Rage-independent autoreactive $B$ cell activation in response to chromatin and HMGB1/DNA immune complexes. Autoimmunity 43: 103-110, 2010.

17. Ding HS and Yang J: High mobility group box-1 and cardiovascular diseases. Saudi Med J 31: 486-489, 2010.

18. Yao HC, Zhao AP, Han QF, Wu L, Yao DK and Wang LX: Correlation between serum high-mobility group box-1 levels and high-sensitivity C-reactive protein and troponin I in patients with coronary artery disease. Exp Ther Med 6: 121-124, 2013.

19. Hashimoto T, Ishii J, Kitagawa F, Yamada S, Hattori K, Okumura M, Naruse H, Motoyama S, Matsui S, Tanaka I, et al: Circulating high-mobility group box 1 and cardiovascular mortality in unstable angina and non-ST-segment elevation myocardial infarction. Atherosclerosis 221: 490-495, 2012.

20. Ulloa L and Messmer D: High-mobility group box 1 (HMGB1) protein: Friend and foe. Cytokine Growth Factor Rev 17: 189-201, 2006.

21. Xu H, Yao Y, Su Z, Yang Y, Kao R, Martin CM and Rui T: Endogenous HMGB1 contributes to ischemia-reperfusion-induced myocardial apoptosis by potentiating the effect of TNF-\&alpha;/JNK. Am J Physiol Heart Circ Physiol 300: H913-H921, 2011.

22. Biscetti F, Ghirlanda G and Flex A: Therapeutic potential of high mobility group box-1 in ischemic inury and tissue regeneration. Curr Vasc Pharmacol 9: 677-681, 2011.

23. Abarbanell AM, Hartley JA, Herrmann JL, Weil BR, Wang Y, Manukyan MC, Poynter JA and Meldrum DR: Exogenous high-mobility group box 1 improves myocardial recovery after acute global ischemia/reperfusion injury. Surgery 149: 329-335, 2011.

24. Hu X, Jiang H, Cui B, Xu C, Lu Z and He B: Preconditioning with high mobility group box 1 protein protects against myocardial ischemia-reperfusion injury. Int J Cardiol 145: 111-112, 2010.

25. National Research Council. Guide for the Care and Use of Laboratory Animals. National Academic Press, 1996.

26. Yao HC, Yang LJ, Han QF, Wang LH, Wu L, Zhang CY, Tian KL and Zhang M: Postconditioning with simvastatin decreases the myocardial injury in rats following acute myocardial ischemia. Exp Ther Med 9: 1166-1170, 2015.

27. Wu YB, Shi LL, Wu YJ, Xu WH, Wang L and Ren MS: Protective effect of gliclazide on diabetic peripheral neuropathy through Drp-1 mediated-oxidative stress and apoptosis. Neurosci Lett 523: 45-49, 2012.

28. Swirski FK, Nahrendorf M, Etzrodt M, Wildgruber M, Cortez-Retamozo V, Panizzi P, Figueiredo JL, Kohler RH, Chudnovskiy A, Waterman P, et al: Identification of splenic reservoir monocytes and their deployment to inflammatory sites. Science 325: 612-616, 2009.

29. Kaminski KA, Bonda TA, Korechi J and Musial WJ: Oidative stress and nuetrophil activation-the two keystones of ischemia/reperfusion injury. Int J Cardiol 86: 41-59, 2002.
30. Zhai Y, Busuttil RW and Kupiec-Weglinski JW: Liver ischemia and reperfusion injury: New insights into mechnisms of innate-adaptive immune-mediated tissue inflammation. Am J Transplant 11: 1563-1569, 2011.

31. Jahangiri A, Leifert WR, Kind KL and McMurchie EJ: Dietary fish oil alters cardiomyocyte $\mathrm{Ca} 2+$ dynamics and antioxidant status. Free Radic Bio Med 40: 1592-1602, 2006.

32. Wang Y, Sun J, Liu C and Fang C: Protective effects of crocetin pretreatment on myocardial injury in an ischemia/reperfusion rat model. Eur J Pharmacol 741: 290-296, 2014.

33. Hool LC. Evidence for the regulation of L-type Ca2+ channnelsin the heart by reactive oxygen species: Mechnism for mediating pathology. Clin Exp Phamacol Physiol 35: 229-234, 2008.

34. Rensing H, Bauer I, Kubulus D, Wolf B, Winning J, Ziegeler S and Bauer M: Heme oxygenase-1 gene expression in pericentral hepatocytes through beta-1 adrneoceptor stimulation. Shock 21: 376-387, 2004.

35. Xu Y, Liu B, Zweier JL and He G: Formation of hydrogen peroxide and reduction of peroxynitrite via dismutation of superoxide at reperfusion enhances myocardial blood flow and oxygen consumption in postischemic mouse heart. J Pharmacol Exp Ther 327: 402-410, 2008.

36. van Dijk A,Krijnen PA, Vermond RA, Pronk A, Spreeuwenberg M, Visser FC, Berney R, Paulus WJ, Hack CE, van Milligen FJ and Niessen HW: Inhibition of type $2 \mathrm{~A}$ secretory phospholipase A2 reduces death of cardiomyocytes in acute myocardial infarction. Apoptosis 14: 753-763, 2009.

37. Matsui Y, Takagi H, Qu X, Abdellatif M, Sakoda H, Asano T, Levine B and Sadoshima J: Distinct roles of autophagy in heart during ischemia and reperfusion: Roles of AMP-activated protein kinase and Beclin 1 in mediating autophagy. Circ Res 15: 914-922, 2007.

38. Wang GL, Jiang BH, Rue EA and Semenza GL: Hypoxia-inducible factor-1 is a basic-helix-loop-helix-PAS heterodimer regulated by cellular tension. Proc Natl Aacad Sci USA 92: 5510-5514, 1995.

39. Jewell UR, Kvietikova I, Scheid A, Bauer C, Wenger RH and Gassmann M: Induction of HIF-1alpha in response to hypoxia is instantaneous. FASEB J 15: 1312-1314, 2001.

40. Zarbin MA: Current concepts in the pathogenesis of age related macular degeneration. Arch Ophthalmol 122: 598-614, 2004.

41. AI-Salam S and Hashmi S: Galectin-1 in early acute myocardail infarction. PloS One 9: e86994, 2014.

42. Rapino C, Bianchi G, Di Giulio C, Centurione L, Cacchio M, Antonucci A and Cataldi A: HIF-1alpha cytoplasmic accumulation is associated with cell death in old rat cerebral cortex exposed to intermittent hypoxia. Aging Cell 4: 177-185, 2005.

43. Siddqi A, Aminova LR and Ratan RR: Hypoxia inducible factor proly 4-hydroxylase encimes: Center stage in the battle against hypoxia, metabolic compomise and oxidative stress. Neurochem Res 32: 931-946, 2007.

44. Wang Z and Si LY: Hypoxia inducible factor-1 $\alpha$ and vascular endothelial growth factor in the cardioprotective effects of intermittent hypoxia in rats. Ups J Med Sci 118: 65-74, 2013.

45. Ke Q and Costa M: Hypoxia-inducible factor-1 (HIF-1). Mol Pharmacol 70: 1469-1480, 2006.

46. Hashmi S and Al-Salam S: Hypoxia-inducible factor-1 alpha in the heart: A double agent? Cardiol Rev 20: 268-273, 2012.

47. Robador PA, San José G, Rodríguez C, Guadall A, Moreno MU, Beaumont J, Fortuño A, Díez J, Martínez-González J and Zalba G: HIF-1 mediated up-regulation of cardiotrophin-1 is involved in the survival response of cardiomyocytes to hypoxia. Cardiovasc Res 92: 247-255, 2011.

48. Blanco Pampín J, García Rivero SA, Otero Cepeda XL, Vázquez Boquete A, Forteza Vila J and Hinojal Fonseca R: Immunohistochemical expression of HIF-lalpha in response to early myocardial ischemia. J Forensic Sci 51: 120-124, 2006.

49. Cai Z, Zhong H, Bosch-Marce M, Fox-Talbot K, Wang L, Wei C, Trush MA and Semenza GL: Complete loss of ischaemic preconditioning-induced cardioprotection in mice with partial deficiency of HIF-1 alpha. Cardiovasc Res 77: 463-470, 2008.

50. Huang Y, Hickey RP, Yeh JL, Liu D, Dadak A, Young LH, Johnson RS and Giordano FJ: Cardiac myocyte-specific HIF-1alpha deletion alters vascularization, energy availability, calcium flux and contractility in the normoxic heart. Faseb J 18: 1138-1140, 2004.

51. Cantley LC: The phosphoinositide 3-kinase pathway. Science 296: 1655-1657, 2002.

52. Wu QL, Shen T, Ma H and Wang JK: Sufentanil postconditioning protects the myocardium from ischemia-reperfusion via PI3k/Akt-GSK-3ß pathway. J Surg Res 178: 563-570, 2012. 
53. Yu Y, Jia XJ, Zong QF, Zhang GJ, Ye HW, Hu J, Gao Q and Guan SD: Remote ischemic postconditioning protects the heart by upregulating ALDH2 expression levels through the PI3K/Akt signal pathway. Mol Med Rep 10: 536-542, 2014.

54. Chen K, Li G, Geng F, Zhang Z, Li J, Yang M, Dong L and Gao F Berberine reduces ischemia/reperfusion-induced myocardial appoptosis via activating AMPK and PI3K-Akt signaling in diabetic rats. Appoptosis 19: 946-957, 2014.

55. Zhou Y, Wang D, Gao X, Lew K, Richards AM and Wang P: MTORC2 phosphorylation of Akt1: A possible mechnism for hydrogen sulfide-induced cardioprotection. PloS One 9: e99665, 2014.
56. Lu C, Ha T, Wang X, Liu L, Zhang X, Kimbrough EO, Sha Z, Guan M, Schweitzer J, Kalbfleisch J, et al: The TLR9 ligand, CpG-ODN, induces protection against cerebral ischemia/reperfusion injury via activation of PI3K/Akt signaling. J Am Heart Assoc 3: e000629, 2014.

57. Zuo W, Chen J, Zhang S, Tang J, Liu H, Zhang D and Chen N: $\mathrm{M}-\mathrm{H} 004$ prevents toxicity induced by delayed treatment of tPA in a rat model of focal cerebal ischemia involving PKA-and PI3Kindependent Akt activation. Eur J Neurosci 39: 2107-2118, 2014.

58. Chen W, Zheng G, Yang S, Ping W, Fu X, Zhang N, Wang DW and Wang J: CYP2J2 and EETs protect against oxidative stress and apopotosis in vivo and in vitro Following Lung Ischemia/Reperfusion. Cell Physio Biochem 33: 1663-1680, 2014. 